\title{
Aneurysmal isolated intracerebral hemorrhage and/or intraventricular hemorrhage without subarachnoid hemorrhage: a rare and perplexing scenario in neurosurgical practice
}

\author{
Guichen $\mathrm{Li}^{1}$, Xiaobo Zhu ${ }^{2}$, Yang Zhang ${ }^{2^{*}}$, Jinchuan Zhao ${ }^{2}$, Xianfeng Gao ${ }^{2}$ and Kun Hou ${ }^{2 *}$
}

\begin{abstract}
Ruptured aneurysms with a presentation of intracerebral hemorrhage $(\mathrm{ICH})$ and/or intraventricular hemorrhage (IVH) without subarachnoid hemorrhage (SAH) are rarely reported. Issues on the clinical characteristics, mechanism, diagnosis, treatment and prognosis of this rare entity are obscure to us. We present two cases of ruptured aneurysms with a presentation of isolated ICH. A systematic review of the literature was also conducted. There were 21 cases plus our cases. Good recovery was achieved in 10 patients (47.6\%). Pertaining to location, $38 \%$ of presenting aneurysms were on the right side, $52 \%$ were on the left side, and $10 \%$ were midline. Anterior circulation aneurysms were identified in $81 \%$ of patients (7 PComA, 8 MCA, 1 ICA, 1 AComA) and posterior in $19 \%$ of patients (3 PCA, 1 BA). Sizes of the aneurysms ranged from $3 \mathrm{~mm}$ to $40 \mathrm{~mm}$ (16.21 \pm 8.45 ). Ruptured aneurysms at the main trunks of the Willis cycle with a presentation of isolated ICH and/or IVH without SAH is extremely rare. The cause of this rare entity is multifactorial. The diagnosis and management of this entity pose a great challenge to us. The prognosis was discouraging based on the now available data.
\end{abstract}

Keywords: Ruptured aneurysm, Intracerebral hemorrhage, Intraventricular hemorrhage, Subarachnoid hemorrhage

\section{Background}

It's well known that ruptured cerebral aneurysms present with subarachnoid (SAH) with or without intracerebral and/or intraventricular extension. But, on rare occasions, an aneurysmal rupture may present with isolated intracerebral hemorrhage (ICH) and/or intraventricular hemorrhage (IVH) without SAH $[1,2]$. According to the largest case series by Thai et al., only $1.6 \%$ (13/822) of patients after aneurysmal rupture presented with isolated $\mathrm{ICH}$ and/or IVH [1]. However, most of the reported cases of aneurysmal rupture with isolated ICH and/or IVH were secondary to aneurysms situated in the deeper or distal cerebral arteries $[2,3]$. As a result of their adjacency to the

\footnotetext{
*Correspondence: zhangyang_930@126.com; houkunscience@163.com ${ }^{2}$ Department of Neurosurgery, The First Hospital of Jilin University, Jilin, China

Full list of author information is available at the end of the article
}

brain surface, ruptured aneurysms at the main trunks of the Willis cycle with a presentation of isolated $\mathrm{ICH}$ and/or IVH are extremely rare $[1,4-8]$.

Here, we present another two cases of isolated ICH due to ruptured intracranial aneurysms at the main trunks of the Willis cycle. In addition, we conducted a systematic literature review to better elucidate the characteristics, mechanism, diagnosis, treatment and prognosis of this rare entity.

\section{Materials and methods}

Two cases of isolated ICH due to ruptured intracranial aneurysms were firstly illustrated. Then a PubMed search of the published peer-reviewed articles on Dec 10th, 2015 was performed. The following key words were used in relevant combinations: ruptured aneurysm, intracerebral/ intraparenchymal hemorrhage, without subarachnoid 
hemorrhage. The reference lists of the identified articles were also manually searched for additional studies.

\section{Definition of main trunk of the Willis cycle}

First and second grade branches of internal carotid artery (ICA), anterior cerebral artery (ACA), anterior communicating artery (AComA), middle cerebral artery (MCA), posterior cerebral artery (PCA), posterior communicating artery (PComA) and basilar artery (BA) that run along the brain surface.

\section{Definition of outcome}

Glasgow Outcome scale (GOS) score on discharge or follow-up $>3$ was deemed good recovery.

\section{Results}

\section{Case illustrations}

\section{Case 1}

A 73-year-old man (nonsmoker) was admitted to the First Hospital of Jilin University (Eastern Division) $1 \mathrm{~h}$ after a sudden onset of mental state disturbance and right hemiparesis. His Glasgow Coma Scale (GCS) score on admission were 11 (E3V3M5). His blood pressure was $182 / 114 \mathrm{mmHg}$ on admission and returned to normal $(<140 / 90 \mathrm{mmHg})$ without antihypertensive agents in $5 \mathrm{~h}$. He had no history of hypertension, diabetes mellitus, anemia, aspirin administration or other systemic diseases. Head computed tomography (CT) on admission showed a left putaminal hemorrhage (Fig. 1a). Further CT angiography (CTA) demonstrated a left MCA aneurysm $(3.1 \mathrm{~mm} \times 1.8 \mathrm{~mm})$ directed superior-posteriorly (Fig. 1b). A causal relationship was suspected between the aneurysm and hematoma.

An emergent microsurgical clipping of the aneurysm combined with hematoma evacuation was planned. Intraoperative exploration found no SAH on the brain surface (Fig. 2a) and the MCA aneurysm was responsible for the putaminal hemorrhage (Fig. $2 \mathrm{~b}$ ). The intraoperative process was uneventful. He developed severe pneumonia
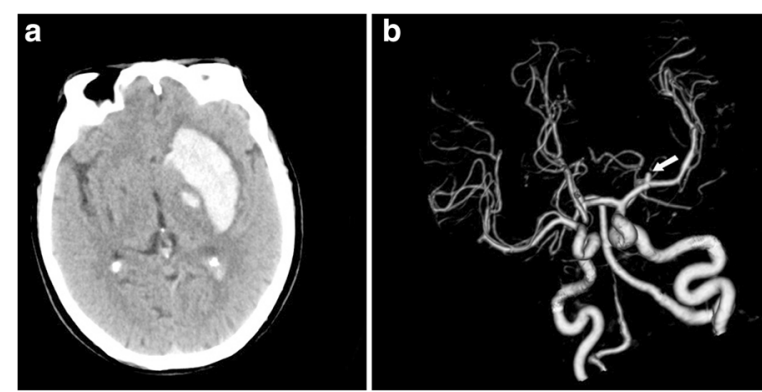

Fig. 1 Head CT shows a left putaminal hemorrhage (a), further CTA demonstrates a left MCA (M1) aneurysm $(3.1 \times 1.8 \mathrm{~mm})$ directed superior-posteriorly (b) and was mechanically ventilated for 13 days postoperatively. He was discharged, with a GCS score GOS score of 13 (E4V3M6) and three separately, 52 days after admission. GOS score was 3 on outpatient follow-up 6 months later.

\section{Case 2}

A 59-year-old man (nonsmoker) was admitted to the First Hospital of Jilin University (Eastern Division) three days after a sudden onset of moderate headache. His Glasgow Coma Scale (GCS) score on admission were 15. His blood pressure, blood glucose, hematocrit and hemoglobin concentration were within normal limit on admission and through his hospitalization. He and his relatives denied history of anticoagulant and antiplatelet agent administration and any family history of SAH and intracranial aneurysm. Head CT on admission revealed a round occupying lesion and perilesional hemorrhage in the right frontal lobe (Fig. 3a). Further CTA demonstrated the occupying lesion to be an aneurysm $(22 \mathrm{~mm} \times 19 \mathrm{~mm})$ at the AComA (Fig. 3b).

An emergent microsurgical clipping of the intracranial aneurysm via right pterional approach was planned. Rupture of the aneurysm was confirmed intraoperatively and no SAH was noticed. The intraoperative process was uneventful. He experienced a favorable postoperative course and was discharged 15 days after admission living independently. Follow-up 1 year later showed that he had experienced a perfect recovery.

\section{Systematic review}

Six studies (five case reports and one case series) of ruptured cerebral aneurysms at the main trunks of the Willis cycle with a presentation of isolated $\mathrm{ICH}$ and/or IVH were identified. There were 21 cases plus our cases (Table 1). Fifty-seven percent (12/21) of the patients were female. The identified patients aged from 14 to 73 years (52.8 \pm 9 , excluding one outlier aged 3 months). Of the 21 patients, definite mention of whether having a history of hypertension or diabetes mellitus or not was found in six patients. Three out of the six patients $(50 \%)$ had a history of hypertension. No history of diabetes mellitus was mentioned in all the patients. Eight-six percent (18/21) of the patients underwent surgical clipping of the responsible aneurysms, $4.7 \%(1 / 21)$ of the patients underwent coiling and $9.5 \%(2 / 21)$ died of too rapid deterioration to further management. GCS score on admission was recorded in 17 patients $(10.64 \pm 3.55)$. Discharge GOS score could be obtained directly or indirectly in 20 patients $(3.35 \pm 1.25)$. Good recovery was achieved in ten patients $(47.6 \%)$. Pertaining to location, $38 \%$ (8) of presenting aneurysms were on the right side, $52 \%$ (11) were on the left side, and $10 \%$ (2) were midline. Anterior circulation aneurysms were identified in $81 \%$ of patients (7 PComA, 8 MCA, 1 ICA, 1 AComA) and posterior in $19 \%$ of patients (3 PCA, $1 \mathrm{BA}$ ). 


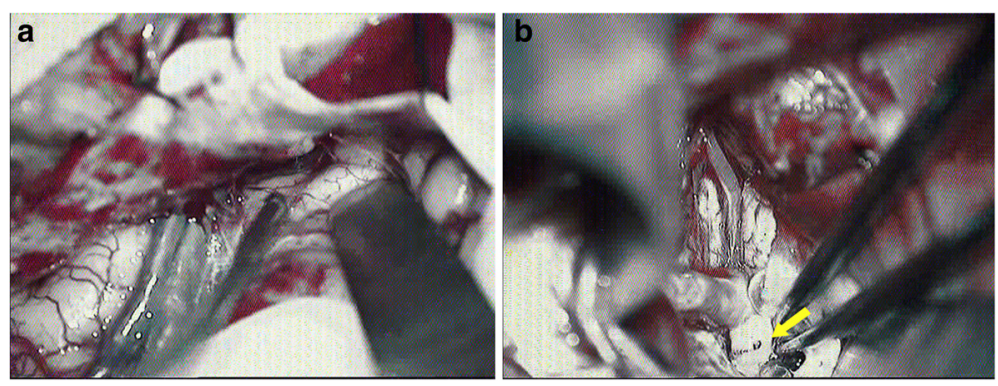

Fig. 2 Intraoperative exploration found no SAH on the brain surface (a) and the dome of the aneurysm is partly buried into the cerebral parenchyma and responsible for the intracerebral hemorrhage (b)

Sizes of the aneurysms ranged from $3 \mathrm{~mm}$ to $40 \mathrm{~mm}$ $(16.21 \pm 8.45)$.

\section{Discussion}

Ruptured aneurysm often presents with $\mathrm{SAH}$ and based on the characteristic CT imaging it's easy to make the diagnosis of aneurysmal SAH (aSAH). However on very rare occasions, ruptured aneurysm could present with isolated ICH and/or IVH without SAH [1, 2, 4-8], which may lead to delayed and incorrect diagnosis and management [9]. Because of their anatomic characteristics, it's understandable that aneurysms at the peripheral cerebral arteries could cause isolated ICH and/or IVH, but aneurysms at the main trunks of the Willis cycle with such presentation is rather obscure [2, 9]. The cause of this rare entity is multifactorial.

Firstly, delayed CT scan after bleeding ictus might lead to false negative result of $\mathrm{SAH}$. The sensitivity of $\mathrm{CT}$ imaging continues to decrease with the development of time in diagnosing SAH $[10,11]$. According to Thai et al.'s case series 6 of the 13 ruptured aneurysms without SAH had reported or suspected sentinel events, averaging 6.3 days before admission [1]. Secondly, the mass effect of ICH could compress the brain parenchyma and makes it denser. ICH could also squeeze out and dilute the blood
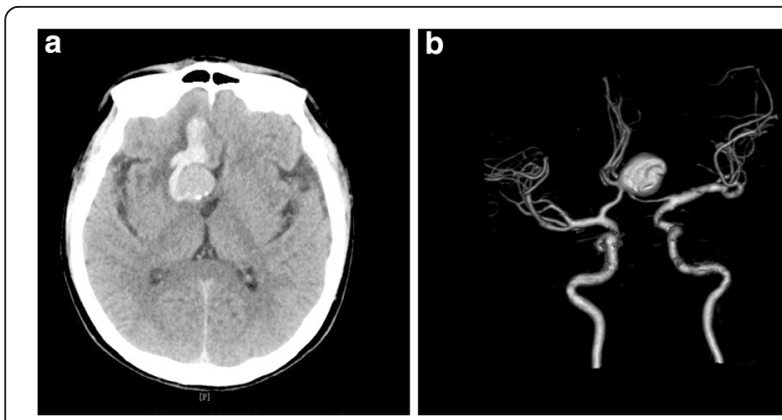

Fig. 3 CT on admission reveals a round occupying lesion and perilesional hemorrhage in the right frontal lobe (a). And some punctate calcification of the lesion is also noted (a). Further CTA demonstrates the occupying lesion to be an aneurysm $(22 \times 19 \mathrm{~mm})$ at the AComA (b) component under the subarachnoid space. The superposition effect of aneurysmal ICH during this process increases the difficulty of diagnosing tiny SAH. Thirdly, dome of an aneurysm buried into the cerebral parenchyma is another cause. The intraoperative findings of one previous case report and our cases had demonstrated and recorded this fact [6]. Our review showed that the average diameter of the aneurysms presented with isolated $\mathrm{ICH}$ and/or IVH was $16.21 \mathrm{~mm}$, which is apparently larger than that of the general intracranial aneurysms. And it's conceivable that larger aneurysms have more chance to be partly buried into the cerebral parenchyma. Furthermore, it's noteworthy that severe acute or chronic anemia is another cause of false negative result. The hyperintensity appearance on noncontrast head $\mathrm{CT}$ is a reflection of electron density, and there is a linear relationship to the hematocrit and hemoglobin concentration [8]. The preliminary statistical analysis of our review showed that $57 \%(12 / 21)$ of the patients were female, which is in accordance with the sex differences in the incidence of aSAH [12]. This might imply that sex does not play an important role in the incidence of this specific entity.

One of the challenges this entity poses to us is correct and timely diagnosis. According to Park J's analysis of 62 patients with spontaneous putaminal hemorrhage, $62.5 \%$ of the younger ( $\leq 55$ years) normotensive patients resulted in angiographic abnormalities (including $1 \mathrm{MCA}$ aneurysm) [5]. So, angiographic modalities should be considered for younger and normotensive patients with spontaneous putaminal hemorrhage. Furthermore, patients presenting with a head $\mathrm{CT}$ scan revealing $\mathrm{ICH}$ in the temporal lobe with or without frontal, parietal, and/or intraventricular involvement should be considered for the possibility of a ruptured aneurysm, even in the absence of diffuse SAH [1]. However, of the identified 21 patients, definite mention of whether having a history of hypertension or not was only recorded in six patients. Three out of the six (50 \%) patients, had a history of hypertension. And 7 of the 21 patients were older than 55 years old. So, patients of isolated ICH and/or IVH with a history of hypertension and older age could not be exempted from ruptured aneurysms. As delayed CT scan 
Table 1 Reports of ruptured aneurysms at the main trunks of the Willis cycle with a presentation of isolated ICH and/or IVH

\begin{tabular}{|c|c|c|c|c|c|c|c|c|}
\hline First author (year) & (Age/sex) & CT finding & Parent artery & Size & $\begin{array}{l}\text { GCS score at } \\
\text { admission }\end{array}$ & $\begin{array}{l}\text { Predisposing } \\
\text { factors }\end{array}$ & Management & $\begin{array}{l}\text { Discharge GOS } \\
\text { score }\end{array}$ \\
\hline \multirow[t]{2}{*}{ Scott BA (1988) [4] } & 54 years/M & $\mathrm{ICH}$ & Right PCA & $25 \mathrm{~mm}$ & NA/NM & NA/NM & Conservative & 1 \\
\hline & 48 years/F & $\mathrm{ICH}$ & Right PCA & $25 \mathrm{~mm}$ & NA/NM & NA/NM & Conservative & 1 \\
\hline \multirow[t]{13}{*}{ Thai QA (2005) [1] } & 62 years/F & $\mathrm{ICH}, \mathrm{IVH}$ & Left PComA & $25 \mathrm{~mm}$ & 9 & NA/NM & Clip & 5 \\
\hline & 49 years/F & $\mathrm{ICH}$ & Left MCA & $15 \mathrm{~mm}$ & 7 & NA/NM & Clip & 4 \\
\hline & 69 years/F & $\mathrm{ICH}$ & Right PComA & $10 \mathrm{~mm}$ & 15 & NA/NM & Clip & 5 \\
\hline & 70 year/F & $\mathrm{ICH}$ & Left MCA & $15 \mathrm{~mm}$ & 12 & NA/NM & Clip & 4 \\
\hline & 46 years/F & $\mathrm{ICH}, \mathrm{IVH}$ & Right MCA & $24 \mathrm{~mm}$ & 8 & NA/NM & Clip & 1 \\
\hline & 58 years $/ M$ & $\mathrm{ICH}$ & Left PComA & $8 \mathrm{~mm}$ & 15 & NA/NM & Clip & 5 \\
\hline & 14 years/F & $\mathrm{ICH}$ & Left P2 & $25 \mathrm{~mm}$ & 15 & NA/NM & Clip & 5 \\
\hline & 3 months/M & $\mathrm{ICH}$ & Left MCA & $40 \mathrm{~mm}$ & 14 & NA/NM & Clip & 5 \\
\hline & 45 years/F & $\mathrm{ICH}$ & Left PComA & $10 \mathrm{~mm}$ & 10 & NA/NM & Clip & 3 \\
\hline & 54 years/F & $\mathrm{ICH}$ & Left PComA & $3 \mathrm{~mm}$ & 15 & NA/NM & Clip & 4 \\
\hline & 51 year/F & $\mathrm{ICH}$ & Right PComA & $12 \mathrm{~mm}$ & 7 & NA/NM & Clip & 3 \\
\hline & 50 year/M & IVH & BA & $24 \mathrm{~mm}$ & 4 & NA/NM & Clip & 2 \\
\hline & 47 years/F & $\mathrm{ICH}, \mathrm{IVH}$ & Right PComA & $6 \mathrm{~mm}$ & 4 & NA/NM & Clip & 1 \\
\hline Park J (2007) [5] & 41 year/F & $\mathrm{ICH}$ & Right MCA Bifurcation & NA/NM & NA/NM & Hypertension & Clip & NA/NM \\
\hline Takeuchi S (2009) [6] & 47 years/M & $\mathrm{ICH}$ & Left MCA Bifurcation & 10 mm & 6 & Hypertension, & Clip & 3 \\
\hline Yamamoto N (2011) [7] & 55 years $/ M$ & $\mathrm{ICH}$ & Left ICA Bifurcation & $6 \mathrm{~mm}$ & 14 & Hypertension & Coil & 4 \\
\hline Matano F (2014) [8] & 64 years/M & $\mathrm{ICH}$ & Right MCA & NA/NM & NA/NM & No & Clip & 3 \\
\hline \multirow[t]{2}{*}{ Present cases } & 73 years/M & $\mathrm{ICH}$ & Left MCA & $3 \mathrm{~mm}$ & 11 & No & Clip & 3 \\
\hline & 59 years/M & $\mathrm{ICH}$ & AComA & $2.2 \mathrm{~mm}$ & 15 & No & Clip & 5 \\
\hline
\end{tabular}

$M$ male, $F$ female, GCS Glasgow Coma Scale, GOS Glasgow Outcome Scale, ICH intrcerebral hemorrhage, $I V H$ intraventricular hemorrhage, $P C A$ posterior cerebral artery, $P C o m A$ posterior communicating artery, MCA middle cerebral artery, $P 2$ posterior cerebral artery (segment 2), BA basilar apex, ICA internal carotid artery, AComA anterior communicating artery, NA/NM not applicable/not mentioned

after bleeding ictus might lead to false negative result of $\mathrm{SAH}$, patients of isolated $\mathrm{ICH}$ and/or IVH who have reported or suspected recent sentinel events should undergone angiographic screening [1]. As average diameter of the aneurysms presented with isolated ICH and/or IVH was apparently larger than that of the general intracranial aneurysms, occupying lesion in the hematoma might be noticed in some cases [4]. Just as our case two illustrated, suspicious occupying lesion in the hematoma warrants further angiographic investigation.

The management of ruptured cerebral aneurysms at the main trunks of the Willis cycle with a presentation of isolated ICH and/or IVH is another challenge both for its urgency in treatment and poorness in prognosis. Perhaps as a result of the mass effect by $\mathrm{ICH}$ and/or IVH, most of the reported cases $(18 / 21)$ underwent urgent surgical clipping of the responsible aneurysms and simultaneous evacuation of the hematoma, except one underwent coiling and the other two died of too rapid deterioration to further management. However, the prognosis was not so encouraging based the now available data. Good recovery was only achieved in ten patients $(47.6 \%)$.

\section{Conclusions}

Ruptured aneurysms at the main trunks of the Willis cycle with a presentation of isolated $\mathrm{ICH}$ and/or IVH without SAH is extremely rare. The cause of this rare entity is multifactorial. The diagnosis and management of this entity pose a great challenge to us. Though angiographic modalities should be considered for younger and normotensive patients with spontaneous putaminal hemorrhage, patients of isolated ICH and/or IVH with a history of hypertension and older age could not be exempted from ruptured aneurysms. The prognosis was discouraging based on the now available data. Ruptured aneurysm at the main trunks of the Willis cycle with a presentation of isolated $\mathrm{ICH}$ and/or IVH is a unique entity and should be treated specifically in future study. More accumulation of similar case series might further elucidate the diagnosis and management of this entity.

\section{Limitations}

This study has several limitations. The available literature is limited to sporadic retrospective study and case reports. There are only a small number of similar cases. As some cases of ruptured aneurysms with only isolated 
ICH and/or IVH might be not distinguished in larger case series, the actual incidence is anticipated to be higher. The time span of this review is so long that the surgical technique and postoperative management might change a lot during this process, which might affect the prognosis greatly among different reports. As a result of the preferred custom in case reporting by different authors, a lot of information is missed concerning the demographic, morphological and follow-up data.

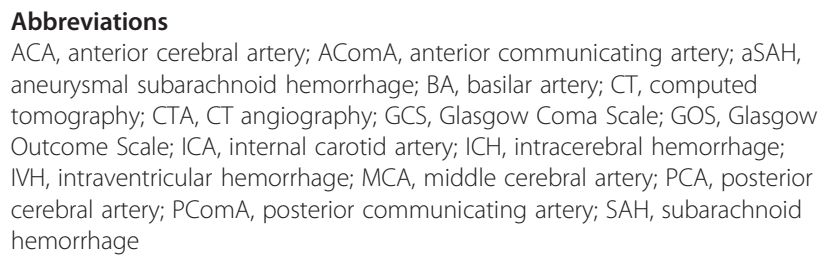

\section{Abbreviations}

ACA, anterior cerebral artery; AComA, anterior communicating artery; aSAH, aneurysmal subarachnoid hemorrhage; BA, basilar artery; CT, computed tomography; CTA, CT angiography; GCS, Glasgow Coma Scale; GOS, Glasgow Outcome Scale; ICA, internal carotid artery; ICH, intracerebral hemorrhage; $\mathrm{IVH}$, intraventricular hemorrhage; MCA, middle cerebral artery; PCA, posterior cerebral artery; PComA, posterior communicating artery; SAH, subarachnoid hemorrhage

\section{Acknowledgement \\ None.}

\section{Funding}

No funding is available.

\section{Availability of data and materials}

Not applicable.

\section{Authors' contributions}

$\mathrm{GL}, \mathrm{KH}$ carried out the studies, participated in collecting data, data interpretation and drafting the manuscript. $Y Z, J Z$ and $X G$ revised the manuscript critically for important intellectual content. $X Z$ helped to draft the manuscript and gave final approval of the version to be published. All authors read and approved the final manuscript. $G L$ and $X Z$ contribute equally to this manuscript and they are co-first authors. $\mathrm{KH}$ and $\mathrm{YZ}$ are co-corresponding authors.

\section{Competing interests}

The authors declare that they have no competing interests.

\section{Consent for publication}

Written informed consent was obtained from the patients for publication of this case report and any accompanying images. A copy of the written consent is available for review by the Editor of this journal.

\section{Ethics approval and consent to participate}

This study was approved by the ethical committee of the First Hospital of Jilin University.

\section{Author details}

${ }^{1}$ Department of Neurology, The First Hospital of Jilin University, Jilin, China. ${ }^{2}$ Department of Neurosurgery, The First Hospital of Jilin University, Jilin, China.

Received: 21 February 2016 Accepted: 10 June 2016

Published online: 18 July 2016

\section{References}

1. Thai QA, Raza SM, Pradilla G, Tamargo RJ. Aneurysmal rupture without subarachnoid hemorrhage: case series and literature review. Neurosurgery. 2005;57(2):225-9.

2. Ahn JY, Cho JH, Lee JW. Distal lenticulostriate artery aneurysm in deep intracerebral haemorrhage. J Neurol Neurosurg Psychiatry. 2007;78(12):1401-3.

3. da Costa LB, Valiante T, Terbrugge K, Tymianski M. Anterior ethmoidal artery aneurysm and intracerebral hemorrhage. AJNR Am J Neuroradiol. 2006; 27(8):1672-4.
4. Scott BA, Weinstein Z, Pulliam MW. Computed tomographic diagnosis of ruptured giant posterior cerebral artery aneurysms. Neurosurgery. 1988;22(3):553-8.

5. Park J, Hwang YH, Baik SK, Kim YS, Park SH, Hamm IS. Angiographic examination of spontaneous putaminal hemorrhage. Cerebrovasc Dis. 2007;24(5):434-8.

6. Takeuchi S, Takasato Y, Masaoka H, Hayakawa T, Otani N, Yoshino Y, Yatsushige $H$, Sugawara T. Case of ruptured middle cerebral artery bifurcation aneurysm presenting as putaminal hemorrhage without subarachnoid hemorrhage. Brain Nerve. 2009;61(10):1171-5.

7. Yamamoto N, Terakawa Y, Okada Y, Mitsuhashi Y, Nishio A, Shimotake K, Murata T. Ruptured internal carotid artery bifurcation aneurysm presenting with only intracerebral hemorrhage without subarachnoid hemorrhage-case report. Neurol Med Chir (Tokyo). 2011;51(2):117-9.

8. Matano F, Murai Y, Nakagawa S, Kato T, Kitamura T, Sekine T, Takagi R, Teramoto A. Atypical radiological and intraoperative findings of acute cerebralhemorrhage caused by ruptured cerebral aneurysm in a patient with severe chronic anemia. J Nippon Med Sch. 2014;81(4):264-8.

9. Cai X, Han S, Feske SK, Chou SH. Pearls and oy-sters: small but consequential: intracerebral hemorrhage caused by lenticulostriate artery aneurysm. Neurology. 2013;80(9):e89-91.

10. van Gijn J, van Dongen KJ. The time course of aneurysmal haemorrhage on computed tomograms. Neuroradiology. 1982;23(3):153-6.

11. Adams Jr HP, Kassell NF, Torner JC, Sahs AL. CT and clinical correlations in recent aneurysmal subarachnoid hemorrhage: a preliminary report of the Cooperative Aneurysm Study. Neurology. 1983;33(8):981-8.

12. Turan N, Heider RA, Zaharieva D, Ahmad FU, Barrow DL, Pradilla G. Sex differences in the formation of intracranial aneurysms and incidence and outcome of subarachnoid hemorrhage: review of experimental and human studies. Translat Stroke Res. 2016;7(1):12-9.
Submit your next manuscript to BioMed Central and we will help you at every step:

- We accept pre-submission inquiries

- Our selector tool helps you to find the most relevant journal

- We provide round the clock customer support

- Convenient online submission

- Thorough peer review

- Inclusion in PubMed and all major indexing services

- Maximum visibility for your research

Submit your manuscript at www.biomedcentral.com/submit
) Biomed Central 